\title{
Uso de la combinación de Propofol-Ketamina para sedación en pacientes
}

\section{sometidos a Cistoscopias.}

Using Propofol-Ketamine Combination for sedating patients undergoing cystoscopies.

C.E.R ${ }^{1}$ Franklin René López $L^{2}$. Ramón Arcadio Amaya L $L^{3}$. María Guadalupe Romero ${ }^{4}$.

\section{RESUMEN}

OBJETIVO: Demostrar la estabilidad hemodinámica, respiratoria en los pacientes que son sometidos a cistoscopia bajo sedación con la asociación de PropofolKetamina.

MATERIAL Y MÉTODOS: El método de obtención de datos fue la encuesta, utilizando la técnica de la entrevista estructurada, con un instrumento que contenía preguntas abiertas y cerradas, de selección múltiple, distribuidas en 2 secciones que incluyeron: Datos generales y Datos clínicos. Los datos se obtenían tanto en el preoperatorio, transoperatorio y postoperatorio.

RESULTADOS: De los pacientes estudiados $48(96 \%)$ no presentaron alteraciones hemodinámicas ni respiratorias y solamente $2(4 \%)$ presentarón cambios hemodinámicos.

CONCLUSIONES: La mezcla de propofolKetamina para sedación no presentarón alteraciones hemodinámicas o respiratorias para todos los pacientes operados ya que su uso es seguro.

Palabras Claves: propofol, Ketamina, analgesia y sedación.

\section{SUMMARY}

OBJECTIVE: to demonstrate the hemodinamic, respiratory and analgesic stability, in patients undergoing cistoscopy under propofol-ketamine sedation.

METHODS:, the data was obtained by a survey, using the technique of a structured interview with an instrument that contained opened-closed questions and multiple choices distributed in two sections: general and clinical data.
The information was obtained pre trans and postoperatively.

CONCLUSIONS: in all patients studied the preparation of ketamina-propofol for sedation in cistoscopies did not altered hemodynamic or respiratory parameters.

Key words: propofol, ketamine, analgesia and sedation.

INTRODUCCIÓN: El anestesiólogo debe proveer al paciente una adecuada y efectiva sedación así como una excelente analgesia. Para lograr estos objetivos se debe proveer un adecuado nivel de sedación mientras se minimiza el dolor y la ansiedad, maximizando la amnesia, minimizando los efectos adversos de las drogas utilizadas, y manteniendo una estabilidad hemodinámica y respiratoria.

El agente farmacológico ideal debería de cumplir con todos estos requisitos, y debería tener un inicio rápido de acción. Ser seguro en todos los grupos de edad, ser barato, y ser igualmente eficaz en múltiples rutas de administración.

Desafortunadamente, en estos tiempos no existe ningún agente único que tenga todas estas cualidades, algunos anestesiólogos tienen que usar combinaciones de diferentes drogas y variando las distintas dosis para lograr muchos de los efectos deseados como sea posible. La más reciente combinación de drogas descrita en la literatura consiste en una pequeña dosis de Ketamina y Propofol (ketofol).

La ketamina fue desarrollada en la década de los 60's como un anestésico seguro y predecible, es un precursor de la fenciclidina, se pueden usar como agente único en procedimientos que requieran

\footnotetext{
1 Comité Editorial de la Revista de Postgrados de Medicina UNAH, Dirección de Docencia e Investigación-HE: Dr. Carlos Vargas Pineda, Dr. Rolando-Aguilera-L, Dra. Guadalupe-Romero-A

2 Residente de 4to. Año Postgrado de Anestesiología, Reanimación y Dolor, UN A H.

3 Jefe del Servicio de Anestesiología, jornada vespertina del Hospital Escuela de Tegucigalpa, Honduras.

4 Medico Máster en epidemiología.
} 
sedación y analgesia, es un anestésico disociativo ya que bloquea la comunicación entre las regiones talamico-limbicos del cerebro. ${ }^{1}$ Provee excelente amnesia y analgesia, y preserva el tono muscular manteniendo los reflejos protectores de la vía aérea y la respiración espontanea. 2,3

El efecto adverso más destacado que aparece por el uso de la ketamina es un fenómeno conocido como delirio de emergencia. ${ }^{4}$ Este ocurre después de algunas horas de la anestesia con ketamina y se manifiesta con confusión, ilusiones y temor. Otros efectos adversos significativos incluyen efectos simpaticomiméticos y vómitos cuando es administrado en dosis para sedación. ${ }^{5}$

El Propofol es un agente hipnótico, sedativo, no barbitúrico, desarrollado en Europa en la década de los 70's y fue gradualmente utilizado por los anestesiólogos en los Estados Unidos alrededor de dos décadas atrás. Su popularidad como agente sedante ha crecido rápidamente debido principalmente a su perfil farmacocinético, su liposolubilidad confiere un rápido inicio y un corto periodo de recuperación, ${ }^{6}$ también tiene las ventajas que funciona como un agente antiemético, un anticonvulsivante y un agente amnésico. ${ }^{7}$ El uso de propofol se ha limitado por una relativamente alta incidencia de hipotensión y depresión respiratoria.7,8,9 Se ha postulado que combinando estos dos agentes para sedaciones, se preserva la eficacia de la sedación mientras se minimizan sus respectivos efectos adversos 10

Los efectos cardiovasculares de cada uno de los fármacos son opuestos, esto teóricamente genera un balance cuando se utilizan juntos. ${ }^{10,11}$

Por todo esto nos hemos visto en la necesidad de realizar un estudio donde se muestre la factibilidad del uso de esta combinación de fármacos y sus principales cambios en la fisiología cardiaca y respiratoria y así beneficiar a todos los pacientes a quienes se les realizará una cistoscopia y que cumplan con los criterios de inclusión.

El propósito del presente estudio es demostrar la estabilidad hemodinámica y respiratoria en los pacientes que son sometidos a cistoscopia bajo sedación con la asociación de Propofol-Ketamina, realizado en el Bloque Medico Quirúrgico del Hospital Escuela durante el periodo comprendido de Julio del 2009 hasta Junio del 2010.

MATERIAL Y MÉTODOS: Se realizó un estudio descriptivo prospectivo. Efectuado Sala de Operaciones del Hospital Escuela, Bloque Medico Quirúrgico, quirófano No 8. Con pacientes que se presentaban para la realización de una cistoscopia, tomando como muestra a todos los pacientes entre los 18 y 70 años en masculino y femenino durante el período comprendido entre Julio del 2009 y Junio del 2010. Se tomo un muestreo a conveniencia de 50 pacientes.

Criterios de inclusión: Pacientes de 18 a 70 años programados para una cistoscopia y con un riesgo anestésico ASA I y ASA II y que desearon participar en el estudio.

Criterios de Exclusión: Pacientes con riesgo anestésico ASA III y ASA IV, historia de alergia al huevo 0 a alguno de los fármacos a utilizar, que no tenga la edad dentro del rango del estudio o que no desee participar en el estudio.

El método de obtención de datos fue la encuesta, distribuidas en 2 secciones que incluyeron: Datos generales y Datos clínicos. Se realizó el estudio desde el momento en que paciente llego a la sala de operaciones, en el quirófano numero 8 , en donde se contaba con el instrumento de trabajo previamente elaborado, luego se continuo con la monitorización del paciente, anotamos los valores hemodinámicos y respiratorios, continuamos con el inicio de la sedación administrando un bolo de la mezcla anestésica de Propofol/Ketamina a

1 Comité Editorial de la Revista de Postgrados de Medicina UNAH, Dirección de Docencia e Investigación-HE: Dr. Carlos Vargas Pineda, Dr. Rolando-Aguilera-L, Dra. Guadalupe-Romero-A

2 Residente de 4to. Año Postgrado de Anestesiología, Reanimación y Dolor, UN A H.

3 Jefe del Servicio de Anestesiología, jornada vespertina del Hospital Escuela de Tegucigalpa, Honduras.

4 Medico Máster en epidemiología. 
una dosis de $1 \mathrm{mg} / \mathrm{kg}$ de peso de ambos fármacos y anotamos nuevamente los valores hemodinámicos y respiratorios, luego al iniciar la cistoscopia se anotaron los valores hemodinámicos y respiratorios cada 5 minutos hasta la finalización del procedimiento quirúrgico. Posteriormente se continúo la valoración en el postoperatorio inmediato y al ser trasladado a la sala de recuperación. Todos los datos se analizaron en el programa Excel XP.

RESULTADOS: En este estudio se incluyeron 50 pacientes, de los cuales el $62 \%(31)$ fueron masculinos y el $38 \%$ (19) femenino; comprendidos entre los 18 y 70 años de edad para ambos sexos, con mayoría en el rango de 40-49 años (56\%) (28).

Del total de pacientes el $96 \%(48)$ no presentaron hipotensión arterial, bradicardia, depresión respiratoria al momento de haberse realizado la sedación, al momento de iniciar la cistoscopia solamente el $4 \%$ de los pacientes estudiados presentaron taquicardia e hipertensión, ninguno de ellos presento depresión respiratoria.

El tiempo mínimo de recuperación fue de 15 minutos y el tiempo máximo de recuperación fue de 19 minutos.

El diagnostico de ingreso fue de Cistitis (14\%), Ca de vejiga (4\%), Hipertrofia Prostática Benigna (8\%), Litiasis ureteral (48\%) y Litiasis vesical (26\%). (Ver Tabla 2).

Tabla 1

Comportamiento hemodinámico.

\begin{tabular}{|c|c|c|c|}
\hline $\begin{array}{c}\text { Presión } \\
\text { Arterial }\end{array}$ & $\begin{array}{c}\text { Inicio de } \\
\text { Cirugía }\end{array}$ & Trans & Fin de Cirugía \\
\hline Hipotensión & 0 & 0 & 0 \\
\hline Normal & 50 & 48 & 48 \\
\hline Hipertensión & 0 & 2 & 2 \\
\hline $\begin{array}{c}\text { Frecuencia } \\
\text { cardiaca }\end{array}$ & $\begin{array}{c}\text { Inicio de } \\
\text { Cirugía }\end{array}$ & Trans & Fin de Cirugía \\
\hline Bradicardia & 0 & 0 & 0 \\
\hline Normal & 50 & 48 & 50 \\
\hline Taquicardia & 0 & 2 & 0 \\
\hline
\end{tabular}

Fuente: instrumento de investigación

De los 50 pacientes estudiados el $96 \%$ mantuvierón su frecuencia respiratoria dentro de los valores normales y solamente el $4 \%$ presentaron taquipnea, ninguno de los pacientes presentó una saturación de oxigeno menor de $95 \%$. (Ver tabla 2 )

Tabla 2

Modificación en la mecánica ventilatoria.

\begin{tabular}{|l|l|l|l|}
\hline $\begin{array}{l}\text { Frecuencia } \\
\text { Respiratoria }\end{array}$ & $\begin{array}{l}\text { Inicio de } \\
\text { cirugía }\end{array}$ & Trans & $\begin{array}{l}\text { Fin de } \\
\text { Cirugía }\end{array}$ \\
\hline $\begin{array}{l}\text { Bradipnea } \\
\text { (menor de 10) }\end{array}$ & 0 & 0 & 0 \\
\hline $\begin{array}{l}\text { Normal } \\
\text { (12 a 17) }\end{array}$ & 50 & 48 & 48 \\
\hline $\begin{array}{l}\text { Taquipnea } \\
\text { (mayor de 18) }\end{array}$ & $\begin{array}{l}\text { Inicio de } \\
\text { Cirugía }\end{array}$ & Trans & $\begin{array}{l}\text { Fin de } \\
\text { Cirugía }\end{array}$ \\
\hline SPo2 & 0 & 0 & 0 \\
\hline Mayor de 96\% & 50 & 50 & 50 \\
\hline De 91 a 95\% & 0 & 0 & 0 \\
\hline Menor de 90\% & 0 & 2 & \\
\hline
\end{tabular}

Fuente: instrumento de investigación

DISCUSIÓN: En nuestro estudio se incluyeron pacientes en las edades comprendidas entre los 18 y 70 años de edad, con predominio del sexo masculino $62 \%$ y $38 \%$ femenino, la litiasis ureteral $(38 \%)$ y la litiasis vesical $(26 \%)$ fueron las patologías que se presentarón con mayor frecuencia a nuestro quirófano. Los resultados obtenidos a nivel hemodinámico y respiratorio se presentaron acorde con lo reportado en la literatura.

El tiempo promedio de la recuperación de los pacientes fue en 17 minutos, el cual concuerda con la literatura.

CONCLUSIÓN: La combinación de Ketamina-Propofol mezcladas en una misma jeringa en un radio de 1:1 parece ser un régimen efectivo de sedación, la recuperación fue mucho más corta y los efectos secundarios fueron mínimos, los pacientes y el equipo quirúrgico salierón satisfechos.

1 Comité Editorial de la Revista de Postgrados de Medicina UNAH, Dirección de Docencia e Investigación-HE: Dr. Carlos Vargas Pineda, Dr. Rolando-Aguilera-L, Dra. Guadalupe-Romero-A

2 Residente de 4to. Año Postgrado de Anestesiología, Reanimación y Dolor, UN A H.

3 Jefe del Servicio de Anestesiología, jornada vespertina del Hospital Escuela de Tegucigalpa, Honduras.

4 Medico Máster en epidemiología. 
AGRADECIMIENTO:

Al Dr.Samayoa por sus invaluables aportes en la realización de esta investigación.

\section{BIBLIOGRAFÍA}

1. Green SM, Krauss B. The semantics of ketamine [editorial]. Ann

Emerg Med. 2000; 39:480-482.

2. Krauss B, Green SM. Procedural sedation and analgesia in children.

Lancet. 2006; 367:766-780.

3. Warncke T, Stubhaug A, et al. Ketamine, an NMDA receptor antagonist,

suppresses spatial and temporal properties

of burn-induced

secondary hyperalgesia in man: a double-

blind, cross-over comparison

with morphine or placebo. Pain. 1997;

72:99-106.

4. Green SM, Rothrock SG, Lynch EL, et al. Intramuscular ketamine for pediatric sedation in the emergency

department: safety profile in 1022

cases. Ann Emerg Med. 1998; 31:688-697.

5. Roback MG, Wathen JE, et al. Adverse

events associated with procedural

sedation and analgesia in a pediatric

emergency department: a

comparison of parenteral drugs. Acad

Emerg Med. 2005; 12:508-513.

6. McLeskey $\mathrm{CH}$, Walawander CA,

Nahrwold, et al. Adverse events in a

multicenter phase IV study of propofol:

evaluation by anesthesiologists

and postanesthesia care unit nurses. Anesth

Analg. 1993; 77:S3-S9.

7. Short SM, Aun CS. Haemodynamic

effects of propofol in children.

Anaesthesia. 1991; 46:783-785.

8. Bassett KE, Anderson JL, et al. Propofol

for procedural sedation

in children in the emergency department.

Ann Emerg Med. 2003;

42:773-782.

9. Hig CC Jr, McLeskey CH, Nahrwold ML,

et al. Hemodynamic effects

of propofol: data from over 25,000 patients.

Anesth Analg. 1993; 77 :
S21-S29.

10. Camu F, Vanlersberghe $C$.

Pharmacology of systemic analgesics.

Best Pract Res Clin Anaesthesiol. 2002; 16:475-488.

11. Morse Z, Sano K, Kanri T. Effects of a propofol-ketamine admixture in human volunteers. Pac Health Dialog. 2003; $10: 51-54$

\footnotetext{
1 Comité Editorial de la Revista de Postgrados de Medicina UNAH, Dirección de Docencia e Investigación-HE: Dr. Carlos Vargas Pineda, Dr. Rolando-Aguilera-L, Dra. Guadalupe-Romero-A

2 Residente de 4to. Año Postgrado de Anestesiología, Reanimación y Dolor, UN A H.

3 Jefe del Servicio de Anestesiología, jornada vespertina del Hospital Escuela de Tegucigalpa, Honduras.

4 Medico Máster en epidemiología.
} 